\title{
Quality of life and its associations with illness perceptions over a 3-month follow-up period in patients with non-small cell lung cancer: A prospective longitudinal study
}

\author{
AYAKO MATSUDA $^{1}$, KUNIHIKO KOBAYASHI ${ }^{2}$, KAORU KUBOTA $^{3}$, YUKARI TSUBATA ${ }^{4}$, KENICHI INOUE ${ }^{5}$, \\ RAJEN RAMAI ${ }^{6}$, JUDITH R. KROEP ${ }^{7}$, MAARTEN J. FISCHER $^{8}$, AD A. KAPTEIN $^{8}$ and KAZUE YAMAOKA ${ }^{9}$ \\ ${ }^{1}$ Department of Hygiene and Public Health, Teikyo University School of Medicine, Tokyo 173-8605; \\ ${ }^{2}$ Department of Mammary Gland Tumor, Saitama International Medical Center, Hidaka, Saitama 350-1298; \\ ${ }^{3}$ Medical Oncology Division, Nippon Medical School Hospital, Tokyo 113-8602; ${ }^{4}$ Department of Respiratory Medicine, \\ Shimane University, Matsue, Shimane 690-0823; ${ }^{5}$ Saitama Cancer Center, Saitama 362-0806, Japan; \\ Departments of ${ }^{6}$ Respiratory Medicine, ${ }^{7}$ Medical Oncology and ${ }^{8}$ Medical Psychology, Leiden University Medical Center, \\ 2333 ZA Leiden, The Netherlands; ${ }^{9}$ Teikyo University Graduate School of Public Health, Tokyo 173-8605, Japan
}

Received May 26, 2021; Accepted September 6, 2021

DOI: $10.3892 /$ wasj.2021.126

\begin{abstract}
In the present prospective longitudinal study with a 3-month follow-up period, the changes in global quality of life (QOL) were investigated over a 3-month period in patients with non-small cell lung cancer (NSCLC). The associations between changes in QOL and illness perceptions were examined. Illness perception groups were classified into two groups by the mean of items of illness perceptions at baseline as follows: 'Strong perception' (strong group) or 'weak perception' (weak group) of the illness. A linear mixed-effects model was used to compare illness perception in the groups in relation to global QOL over a period of 3 months. During the follow-up period, 34 patients were enrolled; 11 patients did not complete the questionnaire on global QOL. Patients in the weak group exhibited a tendency for a lower global QOL over the 3-month period in all illness perception items. The analysis revealed no significant change in global QOL over time in the majority of illness perception dimensions, apart from that related to identity. In this item, the global QOL exhibited a significant difference between the groups over time. The strong group exhibited increased global QOL scores over the 3 -month period than the weak group. Patients who did not have a more sinister view of the illness prior to treatment exhibited a tendency for a decreased global QOL. In addition, patients in the strong perception group had several complaints prior to treatment in the identity item at baseline, and these patients
\end{abstract}

Correspondence to: Dr Ayako Matsuda, Department of Hygiene and Public Health, Teikyo University School of Medicine, 2-11-1 Kaga, Itabashi-ku, Tokyo 173-8605, Japan

E-mail: amatsuda@med.teikyo-u.ac.jp

Key words: quality of life, lung neoplasms, follow-up studies, illness perception had an increased global QOL over the follow-up period. On the whole, the present study demonstrates that the evaluation of QOL may be useful for providing long-term supportive care and may help to address future interventions targeting illness perceptions for patients with NSCLC.

\section{Introduction}

Lung cancer is one of the leading causes of cancer-associated mortality worldwide, and non-small cell lung cancer (NSCLC) accounts for $>80 \%$ of lung cancer histology. Thus, improvements in diagnostics and in the treatment of patients with NSCLC are urgently required (1). Over the past 10 years, notable advancements have been made in the diagnosis and treatment of patients with NSCLC, which have resulted in an improvement in the survival of patients with NSCLC (2). As patients with NSCLC experience both functional restrictions and life-altering symptoms, long-term supportive care is crucial for them (3). Furthermore, recent evidence suggests that providing patients with NSCLC with palliative care not only improves their quality of life (QOL), but also has a positive effect on their survival (4-6).

QOL is an important outcome that should be considered as part of daily clinical oncology practice. The American Society of Clinical Oncology stated that the treatment of metastatic cancer can be recommended even without an improvement in survival if there is an improvement in QOL (7). QOL is an important, yet understudied outcome in patients with NSCLC (8). A previous systematic review of quantitative preference studies of patients with lung cancer confirmed that these patients tended to consider benefit attributes, including QOL, to be more important than other attributes. Additionally, these patients considered overall survival to be the most important (9). QOL is recognized as a relevant end point of growing interest. However, QOL is not included among end points in a sizable proportion of recently published phase III trials on solid tumors (10). Further research is thus required to 
evaluate the treatment effect on QOL in both clinical trials and daily care (11).

'Illness perception' is a concept that refers to the mental representations and personal beliefs that individuals have of an illness, i.e., the cognitions and emotions regarding an illness (12). In a previous study by the authors, it was suggested that addressing illness perception seemed to be a clinically relevant approach in improving the QOL of patients with NSCLC (13). Masson et al (14) suggested the importance of considering illness representations as a determinant of QOL for patients with lung cancer. Changing patient illness perception seems a successful approach in addressing outcomes. For example, in a previous study on patients with myocardial infarction, replacing inadequate illness perceptions by more adaptive ones resulted in an improved functional outcome (15). In the field of NSCLC, the first steps are being taken.

When taking into consideration the assessment of global QOL, it is necessary to recognize that illness perception affects global QOL. This association has been studied in the context of the Common Sense Model, positing that illness perceptions drive coping behavior, which in turn affects QOL (16). In addition, a previous study by the authors on patients with NSCLC in Japan and The Netherlands concluded that illness beliefs affected health-related changes in QOL in individual patients (17). Furthermore, another study detected differences in illness perceptions and QOL between Japanese and Dutch patients with NSCLC (18). What remains unclear in the literature, however, is the association between QOL and illness perceptions and changes in QOL over time.

The present study thus investigated changes in global QOL over a period of 3 months in patients with NSCLC to compare illness perceptions. The associations between QOL changes and illness perceptions were also examined.

\section{Patients and methods}

Study design. From October, 2016 until July, 2019, the present prospective longitudinal study evaluated patients with NSCLC over a 3-month follow-up period at Saitama International Medical Centre, Nippon Medical School Hospital and Shimane University in Japan. Participants completed a case report form that included data such as age, sex, partnership or marital status, and employment at the time of diagnosis; Eastern Cooperative Oncology Group performance status cancer stage (I-III or IV); epidermal growth factor receptor (positive or negative); anaplastic lymphoma kinase (positive or negative); previous surgery (yes or no); previous radiotherapy (yes or no), and total years of smoking. Several questionnaires assessing patient-reported outcomes were administered, and outcomes were assessed at baseline, at 1 month and at 3 months.

Study setting. The study protocol was approved by the Institutional Review Board of Saitama International Medical Center (14-190), Nippon Medical School Hospital (27-07-470) and Shimane University (3567). Written informed consent was obtained from all participants. All study procedures were conducted in accordance with the principles in Declaration of Helsinki and its later amendments.
Patients and eligibility criteria. The eligibility criteria included the diagnosis of NSCLC, an age of $\geq 20$ years, scheduled to receive neoadjuvant or adjuvant chemotherapy, and a physical condition that allowed them to tolerate the investigation. Patients with epidermal growth factor receptor or anaplastic lymphoma kinase mutations, and patients undergoing concurrent radiotherapy and chemotherapy were excluded from the study.

\section{Outcome measures}

Global QOL. The Japanese version of the European Organization for the Research and Treatment of Cancer (EORTC) QOL Questionnaire version 3.0 (EORTC QLQ-C30) was used to assess global QOL. Global QOL was linearly transformed (range, 0-100), with higher scores indicating higher QOL (19).

Illness perception. The Japanese version of the Brief Illness Perception Questionnaire (Brief IPQ) assessed cognitive illness perceptions: Consequences, timeline, personal control, treatment control, identity; emotional perceptions: Concern and emotions; and illness comprehensibility: Coherence (understanding). The Japanese version was adapted from www.uib.no/ipq, Japanese Brief IPQ. To compute the score, the score items of personal control, treatment control and coherence (understanding) are reversed, and are then added to the items of consequences, timeline, identity, concern and emotions according to the Brief IPQ Scoring Instructions from www.uib.no/ipq. All items used a 0-10 scale, with a higher score reflecting a more sinister view of the illness (20). Higher scores indicate strong perceptions in that lung cancer affects the patient's life, has a long duration, can be controlled by their own behavior or medical treatment, involves a number of complaints, elicited concerns, is understandable, and is associated with negative emotions (13).

Data collection and time points. Patients were recruited from three institutions in Japan (Saitama International Medical Centre, Nippon Medical School Hospital and Shimane University). Data were collected at three time points: Baseline at the beginning of the treatment, at $\sim 12$ days after the first round of chemotherapy; at 1 month after the last round of chemotherapy (1 month); and at $\sim 3$ months later (3 months).

Participant characteristics. Sociodemographic and clinical data were obtained from the case report form. Sociodemographic data included age, sex, partnership or marital status, and employment at the time of diagnosis. Clinical data included cancer stage, previous surgery, previous radiotherapy and total years of smoking.

Statistical analysis. The patients were classified into two groups based on the mean of items of illness perceptions at baseline: 'Strong perception' or 'weak perception' of the illness. A linear mixed-effects model was used to compare the two illness perception groups on global QOL over a period of 3 months (21), as previously described. The simple model included global QOL score as the outcome variable. Time parameters were fixed effects: Baseline, 1 month, 3 months; and groups were fixed effects: Strong perception or weak 


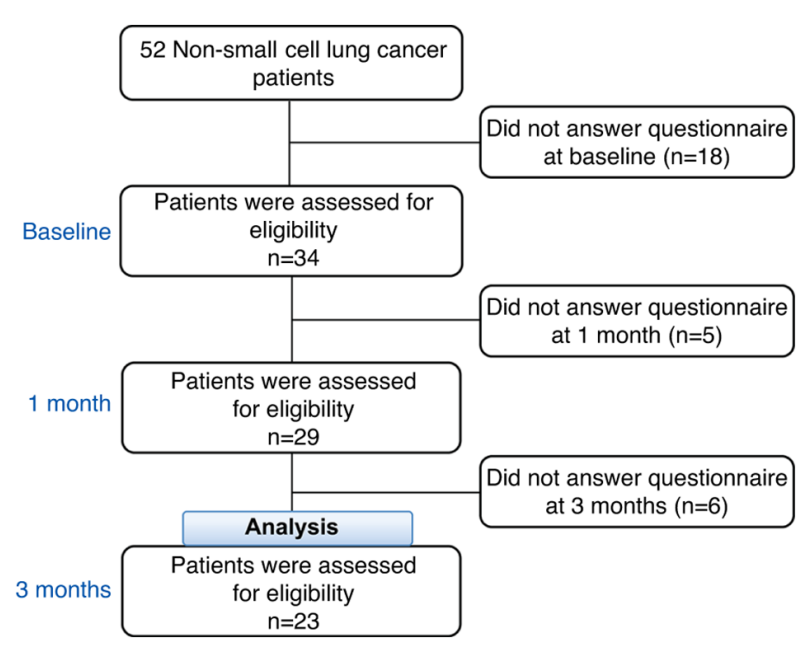

Figure 1. Flow chart of the patients in the present study who were eligible for inclusion.

perception; patient was a random effect; and 'Group x time' was an interaction term. Time was fitted into the model as a categorical variable. The baseline adjusted model included global QOL score as the outcome variable; baseline score for global QOL as a covariate; time and group as fixed effects; patient as random effects; and 'Group x time' as an interaction term. Time was fitted into the model as a categorical variable. Parameter estimates and relative 95\% confidence intervals were obtained (22). Statistical analyses were performed using SAS version 9.4 (SAS Institute, Inc.). $\mathrm{P}<0.05$ (two-sided) was considered to indicate a statistically significant difference.

\section{Results}

Study participants. A flow chart of the patients with NSCLC is presented in Fig. 1. In total, 52 patients were considered eligible. At baseline, 18 patients did not complete the global QOL surveys, leaving 34 patients. At the 1-month follow-up, 5 patients did not complete surveys, leaving 29 patients. At the 3-month follow-up, 6 patients did not complete surveys, leaving 23 patients; these 23 patients were finally analyzed.

Patient characteristics. The characteristics of the participating patients at baseline are presented in Table I. The mean age was 67.2 years, and approximately one-half were male $(52.2 \%)$. The participants included $3(13.0 \%)$ with stage II, 8 (34.8\%) with stage III and 12 (52.2\%) with stage IV cancer. In total, 10 $(43.5 \%)$ patients had a history of surgery, and the majority had never received radiotherapy $(8.7 \%)$. The majority of patients (78.3\%) were married or had a partner, and approximately one-half $(52.2 \%)$ were employed at the time of diagnosis. The mean (standard deviation) values of global QOL and illness perception items of consequences, timeline, personal control, treatment control, identity, concern, understanding and emotional representation at baseline were 67.4 (22.6), 6.1 (2.8), 6.8 (2.5), 4.3 (2.3), 3.2 (1.9), 3.0 (2.6), 7.1 (3.2), 2.8 (1.5), and 3.7 (3.1), respectively. It should be noted that there were missing values as there was no response to the questionnaire at baseline in the illness perception items of timeline and treatment control (missing value: Timeline, $n=1$; treatment control, $n=2$ ).
Table I. Baseline characteristics of the study participants $(n=23)$.

\begin{tabular}{|c|c|c|}
\hline Characteristic & $\begin{array}{c}\mathrm{n} \text { or } \\
\text { mean }\end{array}$ & $\%$ or $\mathrm{SD}$ \\
\hline Age, years, mean (SD) & 67.2 & $(7.2)$ \\
\hline \multicolumn{3}{|l|}{ Sex, n $(\%)$} \\
\hline Male & 12 & $(52.2)$ \\
\hline \multicolumn{3}{|l|}{ Cancer stage, n (\%) } \\
\hline II & 3 & $(13.0)$ \\
\hline III & 8 & $(34.8)$ \\
\hline IV & 12 & $(52.2)$ \\
\hline Previous surgery, n (\%) & 10 & $(43.5)$ \\
\hline Previous radiotherapy, n (\%) & 2 & $(8.7)$ \\
\hline \multicolumn{3}{|l|}{ Total years of smoking } \\
\hline Available data, mean (SD) & 35.8 & $(16.3)$ \\
\hline Missing value ${ }^{\mathrm{a}} \mathrm{n}(\%)$ & 5 & $(21.7)$ \\
\hline \multicolumn{3}{|l|}{ Partnered/married, n (\%) } \\
\hline Available data & 18 & $(78.3)$ \\
\hline Missing value ${ }^{\mathrm{a}}$ & 2 & $(8.7)$ \\
\hline \multicolumn{3}{|l|}{ Employed at time of diagnosis, n (\%) } \\
\hline Available data & 12 & $(52.2)$ \\
\hline Missing value ${ }^{a}$ & 1 & $(4.3)$ \\
\hline Global QOL, mean (SD) $(n=23)$ & 67.4 & $(22.6)$ \\
\hline \multicolumn{3}{|l|}{ Illness perceptions } \\
\hline Consequences, mean (SD) $(n=23)$ & 6.1 & $(3.0)$ \\
\hline Timeline, mean (SD) $(n=22)$ & 6.8 & $(2.5)$ \\
\hline Personal control, mean (SD) $(n=23)$ & 4.3 & $(2.3)$ \\
\hline Treatment control, mean (SD) $(n=21)$ & 3.2 & $(1.9)$ \\
\hline Identity, mean (SD) $(n=23)$ & 3.0 & $(2.6)$ \\
\hline Concern, mean (SD) $(n=23)$ & 7.1 & $(3.2)$ \\
\hline Understanding, mean (SD) $(n=23)$ & 2.8 & $(1.5)$ \\
\hline Emotions, mean (SD) $(\mathrm{n}=23)$ & 3.7 & $(3.1)$ \\
\hline
\end{tabular}

SD, standard deviation; QOL, quality of life. ${ }^{a}$ Missing value indicates that there was no response to the questionnaire.

Global QOL over time comparing strong vs. weak illness perceptions: Follow-up at 3 months. The results of the linear mixed-effects model are presented in Table II. Fig. 2 illustrates global QOL mean scores for the two groups in each item of illness perception over time. Patients in the weak illness perception group exhibited a tendency for a lower global QOL over the period of 3 months in all illness perception items (Fig. 2). No statistically significant differences were observed in the global QOL between the two groups in the majority of the illness perception items, apart from the identity item, which questioned 'How much do you experience symptoms from your illness?' (Table II). For the identity item, the global QOL exhibited significant overall differences over time in the simple model $(\mathrm{P}=0.016)$ and baseline adjusted model $(\mathrm{P}=0.002)$. For the identity item, the strong perception group had increased global QOL scores compared with the weak perception group over the period of 3 months (Fig. 2). 
Table II. Results of the linear mixed-effects model: Estimates of illness perception group differences for global quality of life.

Illness

\begin{tabular}{|c|c|c|c|c|c|c|c|c|}
\hline \multirow{2}{*}{$\begin{array}{l}\text { perception group }^{\mathrm{a}} \\
\text { Consequences }\end{array}$} & \multirow{2}{*}{$\begin{array}{r}\text { Model }^{\mathrm{b}} \\
\text { Simple model }\end{array}$} & \multirow{2}{*}{$\begin{array}{c}\text { Time point } \\
1 \text { month }\end{array}$} & \multirow{2}{*}{$\begin{array}{c}\text { Estimates } \\
13.99\end{array}$} & \multirow{2}{*}{$\begin{array}{l}\text { SE } \\
9.55\end{array}$} & \multicolumn{3}{|c|}{$95 \% \mathrm{CI}$} & \multirow{2}{*}{$\begin{array}{c}\text { P-value }{ }^{c} \\
0.122\end{array}$} \\
\hline & & & & & -5.17 & to & 33.15 & \\
\hline & & 3 months & 20.24 & 10.21 & -0.21 & to & 40.70 & \\
\hline & Baseline adjusted model & 1 month & 13.61 & 8.86 & -4.04 & to & 31.25 & 0.104 \\
\hline & & 3 months & 19.17 & 9.41 & 0.43 & to & 37.91 & \\
\hline \multirow[t]{4}{*}{ Timeline } & Simple model & 1 month & 8.91 & 9.20 & -9.55 & to & 27.37 & 0.409 \\
\hline & & 3 months & 12.64 & 9.99 & -7.39 & to & 32.66 & \\
\hline & Baseline adjusted model & 1 month & 8.12 & 8.42 & -8.66 & to & 24.90 & 0.314 \\
\hline & & 3 months & 13.47 & 9.04 & -4.54 & to & 31.48 & \\
\hline \multirow[t]{4}{*}{ Personal control } & Simple model & 1 month & 2.87 & 9.44 & -16.06 & to & 21.81 & 0.358 \\
\hline & & 3 months & 14.25 & 10.13 & -6.04 & to & 34.54 & \\
\hline & Baseline adjusted model & 1 month & 3.73 & 8.63 & -13.45 & to & 20.92 & 0.243 \\
\hline & & 3 months & 15.39 & 9.23 & -2.98 & to & 33.76 & \\
\hline \multirow[t]{4}{*}{ Treatment control } & Simple model & 1 month & 6.28 & 9.89 & -13.64 & to & 26.20 & 0.140 \\
\hline & & 3 months & 20.97 & 10.46 & -0.08 & to & 42.02 & \\
\hline & Baseline adjusted model & 1 month & 6.08 & 9.23 & -12.34 & to & 24.50 & 0.065 \\
\hline & & 3 months & 22.78 & 9.70 & 3.41 & to & 42.15 & \\
\hline \multirow[t]{4}{*}{ Identity } & Simple model & 1 month & 12.06 & 9.39 & -6.79 & to & 30.91 & 0.016 \\
\hline & & 3 months & 30.21 & 10.12 & 9.91 & to & 50.50 & \\
\hline & Baseline adjusted model & 1 month & 13.19 & 8.50 & -3.72 & to & 30.11 & 0.002 \\
\hline & & 3 months & 33.22 & 9.07 & 15.17 & to & 51.28 & \\
\hline \multirow[t]{4}{*}{ Concern } & Simple model & 1 month & 3.74 & 9.90 & -16.12 & to & 23.59 & 0.463 \\
\hline & & 3 months & 13.55 & 10.91 & -8.30 & to & 35.39 & \\
\hline & Baseline adjusted model & 1 month & 4.70 & 9.08 & -13.38 & to & 22.78 & 0.349 \\
\hline & & 3 months & 14.45 & 9.91 & -5.28 & to & 34.17 & \\
\hline \multirow[t]{4}{*}{ Understanding } & Simple model & 1 month & 4.08 & 9.65 & -15.27 & to & 23.44 & 0.882 \\
\hline & & 3 months & 4.45 & 10.40 & -16.38 & to & 25.28 & \\
\hline & Baseline adjusted model & 1 month & 3.87 & 8.83 & -13.70 & to & 21.44 & 0.882 \\
\hline & & 3 months & 3.87 & 9.45 & -14.93 & to & 22.68 & \\
\hline \multirow[t]{4}{*}{ Emotions } & Simple model & 1 month & 3.42 & 9.57 & -15.78 & to & 22.62 & 0.671 \\
\hline & & 3 months & 9.27 & 10.35 & -11.47 & to & 30.00 & \\
\hline & Baseline adjusted model & 1 month & 3.76 & 8.75 & -13.66 & to & 21.18 & 0.461 \\
\hline & & 3 months & 11.73 & 9.41 & -7.01 & to & 30.47 & \\
\hline
\end{tabular}

Group x time interactions

SE, standard error; QOL, quality of life; CI, confidence interval. allness perception groups were classified into two groups by the mean of

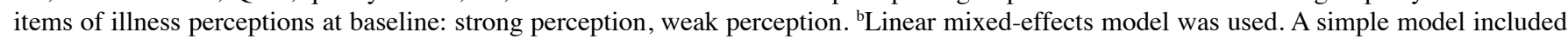
the global QOL score as the outcome variable; 'Time: Baseline, 1 and 3 months', and 'Group: Strong perception, weak perception' as fixed effects; 'patient' as random effects; and 'Group x time' as an interaction term. The baseline adjusted model included the global QOL score as the outcome variable; 'baseline score for global QOL' as a covariate; 'Time: Baseline, 1 and 3 months', and 'Group: Strong perception, weak perception' as fixed effects; 'patient' as random effects; and 'Group x time' as an interaction term. 'Linear mixed-effects model interaction: Time point by group assignment.

\section{Discussion}

In the present study, in Japanese patients with NSCLC, patients who did not have a sinister view of the illness prior to treatment exhibited a tendency for lower global QOL over the period of 3 months (Fig. 2). Additionally, marked differences were observed in the pattern of the global QOL trend between the strong and weak perception groups in the identity item. Patients in the strong perception group in the identity item at baseline had several complaints prior to treatment, and these patients exhibited a markedly higher global QOL over the 3-month period than patients who did not have many complaints (Fig. 2 and Table II).

The adoption of QOL as an end point in oncology clinical trials and the attention given to the timely and complete reporting of QOL results remains limited (10). The findings of the present study may encourage the adoption of QOL as an end point in oncology clinical practice. 

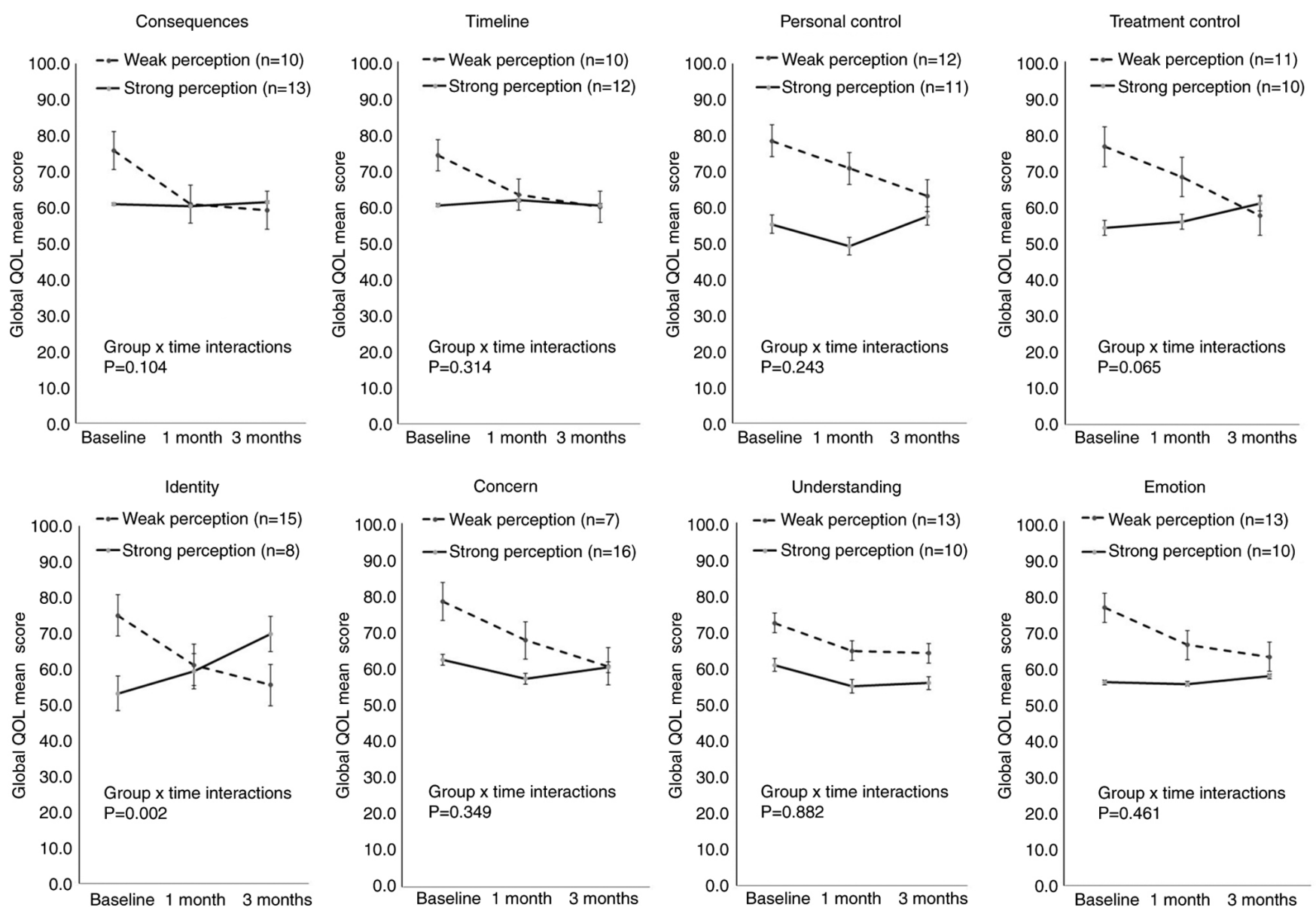

Figure 2. Global QOL mean score for the two groups in each item of the illness perception over time. Error bars represent the standard error of the mean. QOL, quality of life.

Xiao et al (23) demonstrated that patient-reported outcomes (including QOL) were a key information source for assessing clinical benefit in cancer trials and for informing clinical decision making. Significant improvements have been made in incorporating patient-reported outcomes in lung cancer trials (23). However, a previous systematic review reported that QOL results were significantly under-reported, with a disappointing decline in the timely inclusion of results in primary publications (24). Berner et al (25) suggested that an interesting matter for future research was whether health-related QOL could be positively affected by alleviating the perceived threat of the illness and strengthening positive illness beliefs. Hoogerwerf et al (26) demonstrated that the illness perception of patients was not always in line with what the opinions of their physician, illustrating the importance of taking the patient's view into account. The present study demonstrated that the global QOL of patients with NSCLC exhibited a decreasing trend over a period of 3 months if they had a weak illness perception prior to treatment. These results may provide crucial information for a shared decision-making process and may represent a tool to guide medical staff in oncology practice. Additionally, the global QOL differed significantly between the two groups in illness perception for the identity item, and a higher global QOL was observed over the 3-month follow-up period in the strong perception group. A previous study on head and neck cancer survivors described that the illness identity captured patients' perceptions of symptom load (27). In the present study, the patients in the strong identity perception group may have exhibited a higher global QOL over the 3-month period as the patients captured their perceptions of symptom load prior to treatment. These results may prove to be of value to oncology practice.

Kaptein et al (18) reported that accounting for symptoms, illness perceptions and QOL may enhance treatment protocols and outcomes for patients with NSCLC. According to Lehto (28), there is also an urgent need for further research on lung cancer populations that uses baseline psychological distress as an eligibility criterion, given emerging evidence that it may improve the evaluation of intervention benefits over time. The findings of the present study demonstrated that qualifying a patient's illness perception state prior to treatment may help to inform medical staff and researchers on future changes in QOL. It is recommended that clinicians assess the illness perceptions of patients with NSCLC prior to administering treatment and that these patients continuously partake in self-management to improve QOL.

There were some limitations to the present study which should be stated. The sample size was small, as 11 of 34 patients had missing data. However, missing data may not bias the results. There were no marked differences among the 11 ineligible (data not shown: mean age, 65.1 years; $54.5 \%$ cancer stage IV; mean global QOL, 61.4) 
and the 23 eligible patients (mean age, 67.2 years; $52.2 \%$ cancer stage IV; mean global QOL, 67.4). The present study included only patients in Japan. In addition, the mean scores for the treatment control item (3.2), identity item (3.0) and understanding item (2.8) in the Brief IPQ (Table I) were lower than those in the study by Vollmann et al (13) (mean score: Treatment control, 7.7; identity, 5.2; and understanding, 7.5). However, the information about the effect size of global QOL in the present study may prove useful for any plan to calculate sample size. Future international study are required however, for comparison.

In conclusion, the present study suggests that the evaluation of QOL is useful in providing long-term supportive care for patients with NSCLC. The findings presented herein may help to address future interventions targeting illness perceptions of patients with lung cancer with a relatively short mean survival duration.

\section{Acknowledgements}

Not applicable.

\section{Funding}

The present study was supported by the Ministry of Education, Culture, Sports, Science and Technology in Japan Grant-in-Aid for Scientific Research Grant B in 2015 (grant no. 15K19295) and a Grant-in-Aid for Scientific Research Grant C in 2016 (grant no. 16K09562).

\section{Availability of data and materials}

The datasets used and/or analyzed during the present study are available from the corresponding author on reasonable request.

\section{Authors' contributions}

AM was involved in the conceptualization of the study, in analysis using software and data validation. $\mathrm{KKo}, \mathrm{KKu}$ and YT were involved in data collection and supervised the study. $\mathrm{AM}$ and $\mathrm{KKu}$ were involved in funding acquisition. AM, KKo, $\mathrm{KKu}, \mathrm{YT}, \mathrm{KI}, \mathrm{RR}, \mathrm{JRK}, \mathrm{MJF}, \mathrm{AAK}$ and $\mathrm{KY}$ were involved in the study methodology. AM, KKo and AAK were involved in project administration. AM and $\mathrm{KY}$ were involved in the writing of the original draft. KKo, KKu, YT, KI, RR, JRK, MJF and AAK were involved in the writing of the manuscript, and in review and editing. AM and KY confirm the authenticity of all the raw data. All authors have read and approved the final manuscript.

\section{Ethics approval and consent to participate}

The study protocol was approved by the Institutional Review Board of Saitama International Medical Center (14-190), Nippon Medical School Hospital (27-07-470) and Shimane University (3567). Written informed consent was obtained from all participants. All study procedures were conducted in accordance with the principles in Declaration of Helsinki and its later amendments.

\section{Patient consent for publication}

Not applicable.

\section{Competing interests}

The authors declare that they have no competing interests.

\section{References}

1. Reck M,Heigener DF, Mok T, Soria JC and Rabe KF: Management of non-small-cell lung cancer: Recent developments. Lancet 382: 709-719, 2013.

2. Jones GS and Baldwin DR: Recent advances in the management of lung cancer. Clin Med (Lond) 18 (Suppl 2): s41-s46, 2018.

3. Hechtner M, Eichler M, Wehler B, Buhl R, Sebastian M, Stratmann J, Schmidberger H, Gohrbandt B, Peuser J, Kortsik C, et al: Quality of life in NSCLC survivors-a multicenter cross-sectional study. J Thorac Oncol 14: 420-435, 2019.

4. Ambroggi M, Biasini C, Toscani I, Orlandi E, Berte R, Mazzari M and Cavanna L: Can early palliative care with anticancer treatment improve overall survival and patient-related outcomes in advanced lung cancer patients? A review of the literature. Support Care Cancer 26: 2945-2953, 2018.

5. Rutherford C, Campbell R, White K and King M: Patient-reported outcomes as predictors of survival in patients with bowel cancer: A systematic review. Qual Life Res 28: 2871-2887, 2019.

6. Temel JS, Greer JA, El-Jawahri A, Pirl WF, Park ER, Jackson VA, Back AL, Kamdar M, Jacobsen J, Chittenden EH, et al: Effects of early integrated palliative care in patients with lung and GI cancer: A randomized clinical trial. J Clin Oncol 35: 834-841, 2017.

7. Outcomes of cancer treatment for technology assessment and cancer treatment guidelines. American society of clinical oncology. J Clin Oncol 14: 671-679, 1996.

8. Driessen EJM, Janssen-Heijnen MLG, Maas HA, Dingemans AC and van Loon JGM: Study protocol of the NVALT25-ELDAPT trial: Selecting the optimal treatment for older patients with stage III non-small-cell lung cancer. Clin Lung Cancer 19: e849-e852, 2018.

9. Sugitani Y, Sugitani N and Ono S: Quantitative preferences for lung cancer treatment from the patients' perspective: A systematic review. Patient 13: 521-536, 2020.

10. Marandino L, La Salvia A, Sonetto C, De Luca E, Pignataro D, Zichi C, Di Stefano RF, Ghisoni E, Lombardi P, Mariniello A, et al: Deficiencies in health-related quality-of-life assessment and reporting: A systematic review of oncology randomized phase III trials published between 2012 and 2016. Ann Oncol 29: 2288-2295, 2018.

11. van der Weijst L, Surmont V, Schrauwen W and Lievens Y: Systematic literature review of health-related quality of life in locally-advanced non-small cell lung cancer: Has it yet become state-of-the-art? Crit Rev Oncol Hematol 119: 40-49, 2017.

12. Broadbent E, Wilkes C, Koschwanez H, Weinman J, Norton S and Petrie KJ: A systematic review and meta-analysis of the brief illness perception questionnaire. Psychol Health 30: 1361-1385, 2015.

13. Vollmann M, Matsuda A, Kroep JR, Kobayashi K, Kubota K, Inoue K, Yamaoka K, Putter H, Ramai R, Nortier JWR, et al: Illness perceptions and quality of life in patients with non-small-cell lung cancer: A 3-month follow-up pilot study. Patient Relat Outcome Meas 11: 67-71, 2020.

14. Masson N, Dany L, Cannone P, Barlesi F, Baciuchka M and Tomasini P: Illness representations and quality of life in French patients suffering from lung cancer. Psychol Health Med 25: 1119-1129, 2020.

15. Petrie KJ, Cameron LD, Ellis CJ, Buick D and Weinman J: Changing illness perceptions after myocardial infarction: An early intervention randomized controlled trial. Psychosom Med 64: 580-586, 2002.

16. Llewellyn CD, McGurk M and Weinman J: Illness and treatment beliefs in head and neck cancer: Is Leventhal's common sense model a useful framework for determining changes in outcomes over time? J Psychosom Res 63: 17-26, 2007. 
17. van der Kloot WA, Uchida Y, Inoue K, Kobayashi K, Yamaoka K Nortier HW and Kaptein AA: The effects of illness beliefs and chemotherapy impact on quality of life in Japanese and Dutch patients with breast or lung cancer. Chin Clin Oncol 5: 3, 2016.

18. Kaptein AA, Yamaoka K, Snoei L, Kobayashi K, Uchida Y, van der Kloot WA, Tabei T, Kleijn WC, Koster M, Wijnands G, et al: Illness perceptions and quality of life in Japanese and Dutch patients with non-small-cell lung cancer. Lung Cancer 72 : 384-390, 2011.

19. Sprangers MA, Groenvold M, Arraras JI, Franklin J, te Velde A, Muller M, Franzini L, Williams A, de Haes HC, Hopwood P, et al: The European organization for research and treatment of cancer breast cancer-specific quality-of-life questionnaire module: First results from a three-country field study. J Clin Oncol 14: 2756-2768, 1996.

20. Broadbent E, Petrie KJ, Main J and Weinman J: The brief illness perception questionnaire. J Psychosom Res 60: 631-637, 2006.

21. Bonnetain F, Fiteni F, Efficace F and Anota A: Statistical challenges in the analysis of health-related quality of life in cancer clinical trials. J Clin Oncol 34: 1953-1956, 2016.

22. Fitzmaurice GM, Laird NM and Ware JH: Adjustment for baseline response. In: Applied longitudinal analysis. 2nd edition. John Wiley \& Sons, Inc., Hoboken, NJ, pp124-127, 2011.

23. Xiao C, Hurst N and Movsas B: The state of the science in patient-reported outcomes for patients with lung cancer. Semin Respir Crit Care Med 41: 377-385, 2020.
24. Reale ML, De Luca E, Lombardi P, Marandino L, Zichi C, Pignataro D, Ghisoni E, Di Stefano RF, Mariniello A, Trevisi E, et al: Quality of life analysis in lung cancer: A systematic review of phase III trials published between 2012 and 2018. Lung Cancer 139: 47-54, 2020.

25. Berner C, Erlacher L, Fenzl KH and Dorner TE: A cross-sectional study on self-reported physical and mental health-related quality of life in rheumatoid arthritis and the role of illness perception. Health Qual Life Outcomes 16: 238, 2018.

26. Hoogerwerf MA, Ninaber MK, Willems LN and Kaptein AA: 'Feelings are facts': Illness perceptions in patients with lung cancer. Respir Med 106: 1170-1176, 2012.

27. Zhang N, Fielding R, Soong I, Chan KK, Lee C, Ng A, Sze WK, Tsang J, Lee V and Lam WWT: Illness perceptions as predictors of psychological distress among head and neck cancer survivors: A longitudinal study. Head Neck 40: 2362-2371, 2018.

28. Lehto RH: Psychosocial challenges for patients with advanced lung cancer: Interventions to improve well-being. Lung Cancer (Auckl) 8: 79-90, 2017.

(i) (9) This work is licensed under a Creative Commons Attribution-NonCommercial-NoDerivatives 4.0 International (CC BY-NC-ND 4.0) License. 